\title{
Exploiting embedded intelligence in manufacturing decision support
}

\subsection{Introduction}

There have been many advances in the ability to embed intelligence into products and manufacturing equipment in order to collect important data using wireless, intelligent systems of Radio Frequency Identification (RFID) tags and networked sensors [[Xu 14]]. Similarly the ICT industries that support manufacturing businesses continues to expand and develop their range of decision support software across the full range of business requirements from shop floor systems, manufacturing execution systems, enterprise resource planning, product lifecycle management and supply chain management, etc. However, while each of these systems provides important capabilities, the ability to effectively interconnect them in a meaningful trans-disciplinary way is limited [HUB 14] and must be overcome if the visions of Industrie 4.0, 4IR and Smart Manufacturing are to be met.

In the continual need for manufacturing industry to strive for a competitive edge the ICT industry should have the potential to deliver great benefit. Given the potential of ICT a company's multiple decision makers should have ready access to high quality, timely information directed to meet their needs, on which to base critical business decisions. This paper highlights the technological progress that has been made towards meeting this manufacturing requirement and discusses the issues that still need to be resolved.

Chapter written by Paul GOODALL, Heinz LUGO, Richard SHARPE, Kate VAN-LOPIK, Sarogini PEASE, Andrew WEST, Bob YOUNG 


\subsection{Key Technologies}

In this section the key technologies that we consider to be of major importance are highlighted and discussed in turn in terms of their current capabilities for manufacturing decision support. If we start from a decision support perspective the base level requirements are simply to (i) be able to collect the required data, (ii) to direct the appropriate aggregated data to suit the needs of a range of users and (iii) to define data analyse techniques to be able to answer specific sets of multi-user questions. However, to meet these needs is not straightforward and a range of issues

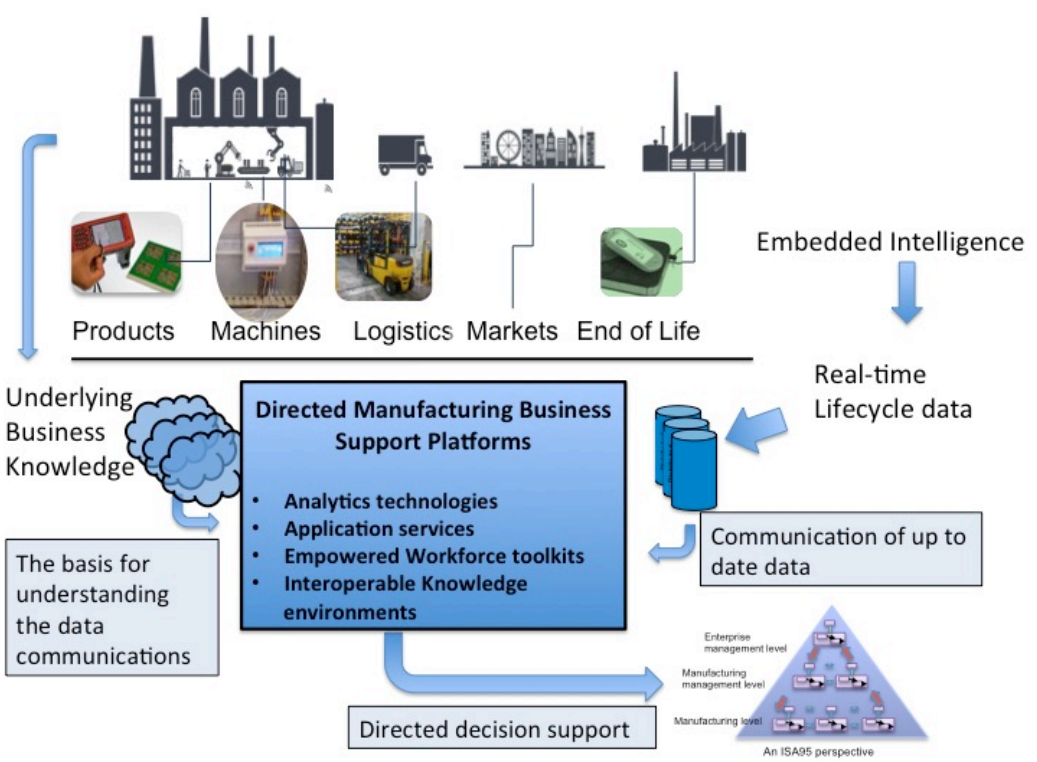

Figure 1. Overview of technologies for directed decision support

needs to be resolved. There is a need to ensure that we can communicate up-to-date high quality information against an understanding of business knowledge across the range of business activities and to be able to build software platforms that can offer a dynamic way of directing information to support the trans-disciplinary needs of multiple users. In addition to the capabilities of embedded components to collect high quality real time data, the main requirements of such a platform are proposed as falling into the following 4 key categories: (i) analytics technologies, (ii) application services (iii) toolkits to empower workers and (iv) interoperable knowledge environments as illustrated in figure 1. Each of these areas are discussed in turn in the following sub-sections. 


\subsubsection{Embedded Systems}

A critical issue for any embedded system is the link between the physical and the cyber world. The robustness of this link determines whether the system can be provided with accurate and timely measurements of the real world. In manufacturing, non-intrusive monitoring is significantly valued as it reduces requirements to pause a production line to maintain or scale infrastructure, avoiding associated productivity losses. Abstracting information from various sources of monitored contextual data, e.g. machine power consumption, tool vibration and asset location, then provides intelligent monitoring, for example to identify which components have been machined by a worn or damaged tool.

The identification of a physical object is the basis of any cyber-physical link and the methods of identification and continuous monitoring need to be appropriate to manufacturing environments i.e. they can operate in harsh environments and be cost effective. Commonly used technologies are passive UHF RFID tags and Wireless Sensor Networks (WSN) which to operate robustly can require a custom design, i.e. selection of appropriate substrate, chip selection, antenna design, and choice of sensors. Once physical objects can be identified the level of intelligence can be extended to aspects such as problem notification e.g. monitoring environmental conditions and decision making e.g. requesting of resources. As a physical object or product becomes more intelligent it requires the capability to access more processing power to be able to extract features from potentially numerous sensors (e.g. position, temperature, acceleration and humidity) and to interpret the results.

An example of an intelligent product's potential output is its location. Asset positioning precision increases with number of sensors, packets sent and traffic rate but is compromised by wireless packet loss that can inevitably be reduced by limiting the wireless transmissions in range of each other [PEA 17A]. At the same time, an architecture of embedded sensors combined with prediction and optimisation models can reduce the need for this type of continuous monitoring [PEA17B]. With an increasing demand on a product to be intelligent the product's demand for power, internal storage and reliable and more frequent communication also increases. As intelligent products become more widespread within manufacturing the requirement for security is also a key concern. 


\subsubsection{Analytics technologies}

Data analytics refers to the process of examining and analysing data with variable types to uncover hidden patterns, correlations and trends. The outcome of this process is to uncover business valuable knowledge in order to increase operational efficiency and explore new market opportunities. Within manufacturing data analytics is often reported as a machine learning solution to a business problem [UHL 17] disregarding both the fact that data analytics exploits knowledge and tools from areas such as data mining, statistical analysis and data modelling.

The usefulness of data analytics is correlated with the time span covered by the gathered data and its analysis. In first instance, the data regarding an immediate snapshot is useful to answer questions regarding what is currently happening. The second instance regards historical data to answer what has happened via the detection of trends and correlations. At this point the understanding of why something happened is not yet achieved through data analytics. The understanding and abstraction of knowledge is the focus of the third instance of data analytics and this is where machine learning and data modelling fit. Once an understanding of why something has occurred has been achieved it is necessary to understand its impact on the business. Tools such as process mining are appropriate at this instance [VAN 07]. To improve and expand the results of data analytics across the range of instances needs an understanding of the increased complexity in the volume of data required, the integration between different data types and sources of data and the improved complexity of the analysis.

\subsubsection{Application Services}

While analytics technologies provide techniques that can be generally applied to identify useful information, application services package these techniques in a reusable and on-going manner to support particular business needs. The process starts from a user application perspective and define services that can support their needs. Examples of the sorts of services that can be defined are product/workpiece traceability, process monitoring, product/workpiece monitoring, logistics monitoring and performance assessment.

For these sorts of services to be effective it is necessary for them to have a clear understanding of the attributes, processes, resources and constraints that they must model and simulate in order to provide useful outputs. This can be challenging within real world manufacturing environments that are highly integrated into a supply chain, dynamic and constantly refining and updating their products, processes and resources. Challenges for application services include the ability to adapt to changing manufacturing environments, ensure they can scale to the needs of production and provide horizontal and vertical interoperability. 


\subsubsection{Empowered workforce toolkits}

An important part of directed decision support is being able to present information to suit the needs of the range of decision makers throughout the business [JAR 17]. While it can be argued that the most important decisions are ensuring that the business makes the correct long-term strategic decisions, these are only effective if the short-term operational decisions are made effectively and the data gathered are representative of the processes being completed. A problem associated with both small and "big" data is one of ensuring veracity [WHI 12]. Veracity may be affected by factors such as choice of sensors or filtering algorithms, and the users of the devices or processes being monitored. This human input can be supported by adopting a user centred approach. When stakeholders are involved in changes they are more likely to adopt new processes and technology and develop the shared mental models that contribute to positive behaviour.

While offering effective graphical user interfaces to suit strategic and tactical level decision makers are important, potentially the most important are those that enable rapid reaction to real time data changes such as anomaly detection. The provision of appropriate real time data may also be used to develop and supplement operator skills and abilities in improved efficiency and reduced operator stress.

The role of augmented reality in empowering the workforce is potentially where rapid benefits can be achieved. These toolkits can enable workers to: access information hands free; exploit digital twins to locate resources, products and people; be made aware of critical manufacturing issues that need immediate attention or maintenance; access on-line resources or be connected with an expert to advise on problem solutions.

\subsubsection{Interoperable knowledge environments}

There is a wide range of trans-disciplinary knowledge and expertise that must be brought together in a successful manufacturing business. Capturing the knowledge of each discipline, the relationships that exist between them, the different semantics that different groups use and ensuring that core knowledge remains secure are a few of the problems that knowledge environments must achieve.

However, a key role for knowledge to be effective is for it to be sharable. It must therefore be captured within an interoperable knowledge environment. However, at the same time, a businesses core knowledge is critical to it's success and needs to be secure so that it is only shared when appropriate.

A great deal of research effort has been targeted at formal ontologies as a route to knowledge interchange but has not produced the flexibility in knowledge base development that is needed. A new approach defining reusable reference ontologies is beginning to show potential [PAL 17] and is being further researched [MOR 17]. 


\subsection{Concluding discussion}

There are clearly huge business benefits to be gained from providing decision makers with high quality, accurate and timely information on which to base their decisions and inform their actions. Each of the areas mentioned above needs to be improved and enhanced for the full range of manufacturing business users to benefit.

At a simple level real-time data can be communicated effectively. However we are far short of the understanding needed to offer up the multiple different aggregations of information needed to satisfy the needs of trans-disciplinary business personnel. Just as importantly the software platforms that start to offer solutions must be dynamically reconfigurable to match the rapid change requirements of manufacturing business.

\subsection{References}

[HUB 14] Huber A. (2014) Presentation by Siemens CEO, World Manufacturing Forum, Milan, July 2014. http://www.ims.org/2014/07/world-manufacturing-forum-2014/

[JAR 17] Jardim-Goncalves, R., Romero, D., \& Grilo, A. (2017). Factories of the future: challenges and leading innovations in intelligent manufacturing. International Journal of Computer Integrated Manufacturing, 30(1), 4-14.

[MOR 17] Morris KC., Kulvatunyou S. (2017) http://blog.mesa.org/2017/03/workingtowards-industrial-ontology.html

[PAL 17] Palmer C, Usman Z, Canciglieri Junior O, Malucelli A, Young RIM (2017) Interoperable manufacturing knowledge systems. International Journal of Production Research 1-20 Oct 2017, doi: 10.1080/00207543.2017.1391416

[PEA 17A] Pease, S. G., Conway, P. P., \& West, A. A. (2017). Hybrid ToF and RSSI realtime semantic tracking with an adaptive industrial internet of things architecture. Journal of Network and Computer Applications, 99(August 2016), 98-109.

[PEA 17B] Pease, S. G., Trueman, R., Davies, C., Grosberg, J., Yau, K. H., Kaur, N., ... West, A. (2018). An intelligent real-time cyber-physical toolset for energy and process prediction and optimisation in the future industrial Internet of Things. Future Generation Computer Systems, 79, 815-829.

[UHL 17] Eckart. Uhlmann, Abdelhakim. Laghmouchi, Claudio. Geisert, Eckhard. Hohwieler. Procedia (2017). Decentralized Data Analytics for Maintenance. Industrie 4.0. Manufacturing, Vol. 11, 2017, Pages 1120-1126.

[VAN 07] W.M.P.van der Aalst, H.A.Reijers, A.J.M.M.Weijters, B.F.van Dongen, A.K.Alves de Medeiros, M.Song, H.M.W.Verbeek (2017) Business process mining: An industrial application.

[WHI 12] White, M. (2012). Digital workplaces: Vision and reality. Business Information Review, 29(4), 205-214. http://doi.org/10.1177/0266382112470412

[XU 14] Xu, L., He, W., Li, S., 2014. Internet of things in industries: a survey. IEEE Trans. Ind. Inform. (4), 1-11. 